\title{
SmartMote: Energy and VoI Aware Solar-Powered Sensor Network Design for Environment Monitoring
}

\author{
Shao-Jie Tang ${ }^{\dagger}$, Cheng $\mathrm{Bo}^{\dagger}$, Xiang-Yang $\mathrm{Li}^{\ddagger \dagger}$, Yunhao $\mathrm{Liu}^{\S}$ Xiaohua Xu${ }^{\dagger}$ Jing Yuan \\ ${ }^{\dagger}$ Department of Computer Science, Illinois Institute of Technology, USA \\ ${ }^{\ddagger}$ The Key Laboratory of Embedded System and Service Computing, Ministry of Education, Tongji University, China. \\ $\S$ TNLIST, Tsinghua University \\ ${ }^{b}$ Department of Computer Science, University of Illinois at Chicago
}

\begin{abstract}
Due to advances in low power micro-sensor technology, energy harvesting techniques, we can now build large scale solar-powered sensor networks to support long-running operations. Solar powered sensors often harvest variable amounts of energy in different weather conditions. Then a primary requirement for an efficient and a long-running solar-powered sensor system is to adapt to changing environment conditions and resources, and to gather as much valuable data as possible. Sensing and collecting data at a constant rate, without taking into account energy availability or data deliverability, will either drain the battery or waste resources. In this work, we design and test a highly efficient and robust solar-powered system SmartMote; and we further present an energy and value of information (VoI) aware routing strategy, that balances the rates of sensing with packet delivery for SmartMote. SmartMote achieves fairness and near maximum utility across the network. We deploy SmartMote in a forest with 100 sensors in order to monitor the humidity, temperature and luminance intensity. Our experimental results corroborate our design.
\end{abstract}

Index Terms - solar-powered, system design, sensor, routing.

\section{INTRODUCTION}

In this paper, we introduce a solar powered sensor systems, SmartMote. In this system each sensor nodes is able to harvest environmental energy e.g., solar power, to support its activity. We test our system in a forest to monitor the environmental changes in the forest. We let this system continuously collect environment information (e.g. temperature, humidity and luminance intensity) and transmit them back to the sink node. The main challenge in developing such systems is to design low-cost and reliable solar-powered sensor mode and dynamic network planning to cooperate with the platform in order to maximize the system utility. Here the system utility is defined as the total value of collected information. Obviously, collecting data at a constant rate in a solar powered sensor system, without considering energy availability, will either drain the battery or waste collected energy. We therefore develop a highly efficient solar-powered system; and present a networking planning strategy to balance the sensing rate with packet delivery for SmartMote. SmartMote is able to achieve fairness and maximum value of collected information across the network.

Many works have recognized the importance of rechargeable batteries in prolonging the sensor system lifetime [1]-
[6]. However, it is still a challenge task to develop low-cost and efficient solar-powered sensor mote in order to support long term and reliable operation. This work aims to propose jointly test bed design and network planning strategy to enable sustainable long running large scale sensor system. The main contribution of this work can be summarized as follows:

- We design a Dual Power Supply system for each sensor, which can automatically switch the input power between solar-powered Li-ion battery and backup AA battery based on real-time charging profile. It enables our system be stable to most environmental noise.

- We propose an enhanced platform, which is controlled by software running on the mote, to maximize charging efficiency. Powered by software-defined charging voltage, our system is able to harvest energy even under harsh environment, e.g., cloudy day.

- We introduce a new concept, value of information (VoI), in this work to evaluate the importance of collected information. We assign different weights to different sensors in order to prioritize the sensing rate of each sensor. By maximizing the overall weight, we are able to achieve maximum value of collected information.

- We implement our platform and network planning strategy in a large scale testbed with more than 100 sensors, and our experimental results show that SmartMote performs pretty well in practice.

This paper is organized as follows: In Section II we briefly demonstrate the test bed. We introduce the system model and problem formulation in Section III. In Section IV and Section V, we describe the platform design. In Section VI, we develop a set of network planning strategies. We evaluate the performance of our system in Section VII, review some related works in Section VIII and concludes this paper in Section IX.

\section{Testbed Demonstration}

We first briefly introduce the testbed used in this work ${ }^{1}$. We adopt the TelosB Mote [7] with a MSP430 processor and CC2420 transceiver. A photodiode (Hamamatsu S1087 series)

\footnotetext{
${ }^{1}$ This work is a part of GreenOrbs project, and the testbed used here may also be used in other sub-projects of GreenOrbs.
} 
on the mote perceives the illuminance. We have two types of motes. The first type of mote is equipped with a (solar powered) rechargeable Li-ion battery and $2 \mathrm{AA}$ batteries, why the second type of motes only have batteries. The detailed design of each sensor node will be demonstrated in Section IV. Our testing composed of two stages: 1) a smaller testbed composed of 10 solar-powered rechargeable nodes for studying charging patterns; and 2) a larger testbed composed of carefully deployed 120 sensors in the forest to monitor the illuminance, temperature and humidity, used to verify several scheduling methods. And we further deploy 5 sink nodes near the road in order to collect the data from the network periodically. We already put a user interface online, interested readers may access http://orbsmap.greenorbs.org for realtime information.

Enclosure. As the system is deployed for long-term monitoring in the forest without human supervision, the sensor nodes must be firmly protected to resist possible weather and physical destruction. The sensor mote is enclosed by a firm weatherproof plastic box with a transparent upper face so that the enclosure has little influence on the illuminance perceived by the sensor.

Software. The sensor program is developed based on Tiny OS 2.1. The Hamamatsu S1087 ParC component provides the illuminance readings, while the Sensirion Sht11C component provides the temperature and humidity readings. The VoltageC component is used to read data from the MCU-internal voltage sensor. The Low Power Listening interface is used to enable low power listening on duty-cycled nodes.

Deployments. The system is deployed on the campus of Zhejiang Forestry University, Hangzhou, China $\left(30^{\circ} 15^{\prime} 28^{\prime \prime} \mathrm{N}\right.$, $119^{\circ} 43^{\prime} 45^{\prime \prime E}$ ), which is about $20,000 \mathrm{~m}^{2}$. We have carried out multiple rounds of deployments for the prototype. The first deployment included 50 nodes, which commenced in July 2008, and lasted for a month. The CC2420-DEF-RFPOWER was set at 31 and the diameter of the resulting network was 6 hops. The second deployment started in early March 2009 and initially included 120 nodes. The CC2420-DEF-RFPOWER was set at 28 and the diameter of the resulting network was 10 hops. The deployment area belongs to the north subtropical monsoon climate, with the annual average temperature $15.3 \sim$ $15.9^{\circ} \mathrm{C}$ and the annual average precipitation $1286 \sim 1424$ $\mathrm{mm}$.

\section{System Model And Problem Formulation}

The aim of this paper is to design and implement a large scale solar-powered sensor network for long term monitoring and data gathering. Basically, our work is composed of two parts: 1) Platform design and 2) Network planning. In the platform design part, we will introduce the detailed design of our planform, e.g., the architecture, the overall design principle, each component and its functionality. Accordingly, we propose a set of network planning strategies in order to collect as much valuable data as possible from the network to the sink nodes. Typically, a network planning strategy specifies

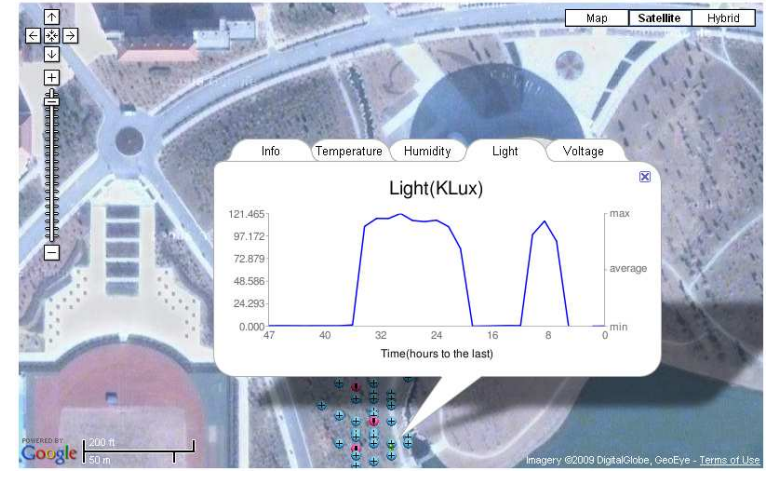

Fig. 1. Illuminance information collected from sensor16.

the sensing rate, transmission rate of each sensor node, the routing strategy and the link scheduling strategy.

Without loss of generality, we assume that there is a set of $n$ sensor nodes deployed over a two-dimensional area. In addition, there are $m$ sink nodes in order to collect the data from the network. The locations of the sensors are fixed and known a priori. We assume that each sensor can perform sensing at certain rate. And they further transmit the sensed data to one of the sink nodes through single- or multihop routing. Each sensor $i$ is equipped with a solar panel which is able to harvest the energy from the environment. A preliminary, and essential, assumption underlying our network planning strategy is that we can estimate the amount of harvested energy in the near future. As we may know, the available energy that can be harvested from the solar panel heavily depends on the environment condition. As shown in Fig.1, even for the same sensor, the charging pattern(in terms of luminance intensity) may vary significantly within one day. For the sake of accuracy, it is necessary for us to monitor the charging pattern frequently in order to obtain an accurate estimation for the near future.

In this work, we define the scheduling period (referred from now on as period) as a time duration of 45 minutes. We let each sensor measure the charging voltage at the beginning of each period through certain hardware mechanisms. By assuming that the charging pattern will not change much through one scheduling period, we can further estimate the total amount of harvested energy during this period.

Assume that sensor $i$ can harvest $E_{i}$ amount of energy during certain period, we next model the energy consumption for data transmission, reception and sensing, based on the first order radio model [8]. Basically, a sensor consumes $\epsilon_{\text {elec }}=50 \mathrm{~nJ} / \mathrm{bit}$ to run the transmitter or receiver circuity, $\epsilon_{a m p}=100 \mathrm{pJ} / \mathrm{bit} / \mathrm{m}^{2}$ for the transmitter amplifier and $\epsilon_{\text {sens }}=70 \mathrm{pJ} /$ bit to generate the sensed data. By assuming one packet contains $k$-bits, the energy consumed by a sensor $i$ in receiving 1-packet data is given by,

$$
R_{X_{i}}=\epsilon_{\text {elec }} \times k .
$$

The energy consumed in transmitting one packet data to sensor 
$j$ is given by, when $d_{i, j}$ is the distance between nodes $i, j$,

$$
T_{X_{i, j}}=\epsilon_{\text {elec }} \times k+\epsilon_{a m p} \times d_{i, j}^{2} \times k
$$

The energy consumed from generating 1 data-packet is

$$
S_{X_{i}}=\epsilon_{\text {sens }} \times k
$$

Based on our experience on deploying large scale sensor network in the forest, we find that different sensors may play different roles on reporting certain specific data. For example, for the sensors deployed nearby bamboo, we expect them to report the humidity information more frequently than the others since humidity information is very essential for the research on bamboo, compared with other plants. In this sense, the value of the sensed data from those sensors are relatively higher than the others in this example. Motivated by this intuition, we tend to assign a higher sensing rate to those "valuable" sensors to ensure the overall value of collected information. In this work, by allowing each sensor to adaptively adjust its sensing rate, we potentially introduce a source of inconsistency in the system, because the criteria by which the scheduler allocates the energy between sensing and transmission for each sensor might be in contrast with that used by the scheduler, which are usually related to value of information ( $\mathrm{VoI}$ ) requirements and fairness. We quantify this issue by defining a weight $w_{i}$ to be associated to each sensor $i$. The weight is set by the scheduler, and it is used to prioritize the sensing rate of each sensor when designing network planning strategy. The sensor which is expected to have higher value deserves a higher sensing rate, further deserves a larger weight. In the previous example, we may assign larger weight to the sensors nearby bamboo than the others. Let $s_{i}$ packets/period denote the sensing rate of sensor $i$ at certain period, and we define the weight gained by successfully transmitting $s_{i}$ amount of data to one of the sink nodes as $w_{i} \times s_{i}$. Then beyond the platform design part, the objective of this work is to find a network planning strategy in order to maximize the overall weight

$$
\sum_{i=1}^{n} w_{i} \cdot s_{i}
$$

of each period.

\section{INITIAL PLATFORM DESIGN}

To store harvested energy, researchers suggested using rechargeable batteries, such as $\mathrm{NiCAD}, \mathrm{NiMH}$, or Li-ion. In our platform, we choose Li-ion as the main battery due to the following reasons: Li-ion batteries can be formed into a wide variety of shapes and sizes so as to fit in various devices. Meanwhile, Li-ion batteries are lighter than the other energyequivalent secondary batteries. The key advantage is the high open circuit voltage that can be obtained in comparison to other rechargeable batteries, such as NiCAD, NiMH. Furthermore, Li-ion batteries do not suffer from memory effect. They also have a low self-discharge rate of approximately $0.1 \%$ per month, compared with over $30 \%$ per month in common NiMH batteries and $10 \%$ per month in NiCAD batteries. Finally, Liion battery owns a higher power density than NiCAD and

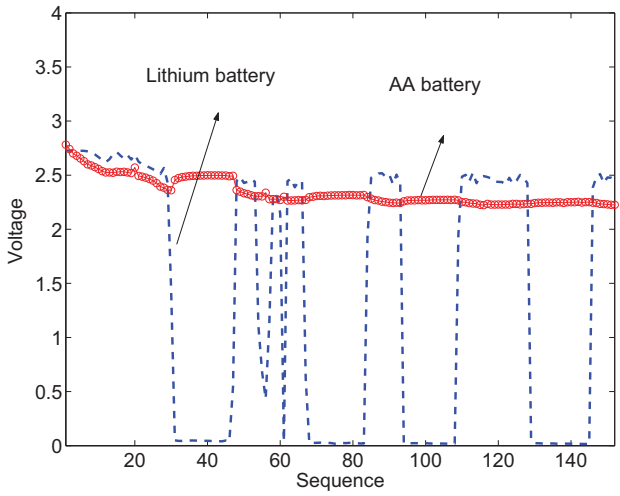

Fig. 3. When the system detects that the voltage of Li-on battery is below $2.7 \mathrm{~V}$, it will switch the input power to AA battery in order to maintain the level of charging voltage.

$\mathrm{NiMH}$ batteries. Some researchers claim that Li-ion battery has only 500 charge cycles, which does not comply to the requirement of sustainable energy supply. However, in our real experiment, it consumes only $1 / 5$ recharge cycle each day through carefully designed network planning strategy, which implies the battery can survive $500 \times 5$ days(more than 5 years). It clearly can meet the requirements in most scenarios.

To ensure the efficiency of our network planning strategy, it is critical to estimate the amount of harvested energy in the near future accurately. As we know, the amount of energy that can be harvested through solar panel heavily depends on the environmental condition, e.g., weather condition. In this work, we periodically collect the charging pattern from each sensor at the beginning of each period(45 minutes), based on which we further estimate the amount of energy that can be harvested during this period. These estimation will be used later as the input of network planning, e.g., energy allocation, for this period. Clearly, if the charging pattern for each sensor remains(or almost) the same through the coming period, e.g., the light strength does not change much, we may expect a acceptable accuracy on the estimation made at the beginning. Unfortunately, there often exists various environmental noises which may affect the charging pattern during this 45 minutes. For example, the cloud may move from one area to the other along with the wind, then the sensors that fall inside the shadow area may not harvest as much energy as estimated. It will further affect the performance of our network planning.

Dual Power System. To make our system more resistance to such environmental noise, we introduce 2 AA batteries as backup power, they will be triggered when the Li-ion battery run out of energy without completing its task within one period. Basically, our system is able to automatically switch the input power to AA battery, as soon as it detects that the charging voltage is not able to sustain the original network planning. Please see Fig. 3 as a illustration.

As shown in Fig. 2(a) our platform comprises five components: (1)the solar panel for energy harvesting; (2)energy storage medium such as Li-ion battery and AA battery; (3)power switch circuit to automatically change the input of 


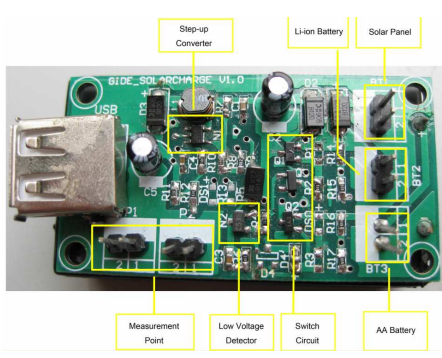

(a)

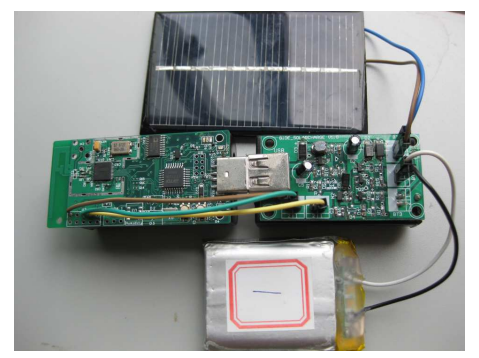

(b)

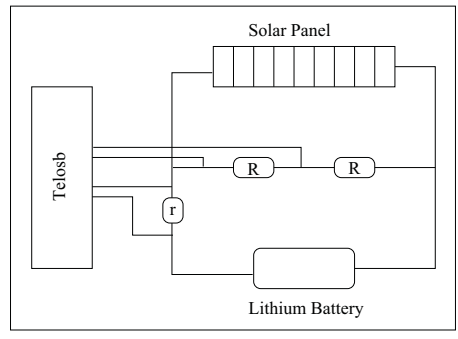

(c)

Fig. 2. (a)The Printed Circuit Board of SmartMote; (b)The Prototype system of SmartMote; (c)Energy Monitoring System Architecture

mote; (4)the power supply circuit with a DC/DC converter for powering the external working sensor node attached to the SmartMote, and (5)the power measurement circuit. The power circuit component plays the most important role in the whole system. It integrates a DC/DC converter and a low voltage detector. The converter is a high-efficient device for supplying a stable and sustainable power for the external sensor node from Li-ion, AA battery or the solar panel. Moreover, a low voltage detector is used to detect both the solar panel and the Li-ion voltages. Coordinating with peripheral circuit, when the voltage of Li-ion battery drops to $2.7 \mathrm{~V}$ and the solar panel cannot provide enough energy, the low voltage detector pulls the relevant pin to ground, so that the peripheral circuit switches the input of SmartMote from Li- ion battery and solar panel to AA battery. On the other hand, when the voltage of the Li-ion battery rises to $2.7 \mathrm{~V}$, the low voltage detector pulls the relevant pin to high, so that the peripheral circuit switches the input of SmartMote from AA battery to Li-ion battery. Readers may doubt why we choose 2 AA batteries but not other rechargeable batteries as backup power. Here we have three main reasons: (1) the voltage of AA battery is relatively stable which is of critical for long term continuous monitoring; (2) the cost of the whole platform can be reduced moderately; (3) in certain harsh environment or weather, the solar energy harvested by panel is deficient, and the rechargeable battery cannot be fully charged, AA battery is able to ensure the reliability of the whole network.

Energy Monitoring Scheme. Remember that in order to estimate the total amount of energy harvested at each period, we need to first measure the charging voltage at the beginning of each period. Then the next challenging problem face us is: How can each sensor measure the charging voltage as well as the voltage of the remained energy in Li-ion battery more accurately and efficiently?

Oscilloscopes have been widely used to measure the energy consumption profile for sensor nodes. However, in large-scale sensor networks, it is difficult to setup an oscilloscope for each sensor node to measure its energy profile. To monitor the realtime condition of the Li-ion battery for each sensor node, we add two high-value resistors $R$ and one low-value resistor $r$ to our SmartMote system as shown in Fig. 2. By measuring the voltage of one high-value resistor and one low-value resistor, we can immediately obtain the voltage and current of the Li- ion battery. We can directly get the voltage through ADC12 port on TelosB. After obtaining the voltage and current, the TelosB node will pack those values and send it back to the sink node by one- or multi-hop, periodically.

\section{Enhanced Platform Design}

In Section IV, we introduce the initial design of the platform in our system. However, when we test our platform in the real experiment, a number of serious practical issues arise. Existing solar energy harvesting solution for wireless sensor nodes usually envisage a simple on/off-threshold charge mechanism relying on a diode connecting the solar panel with the rechargeable battery. Unfortunately, in a diode-based solution, the necessary condition for charging is that the available voltage supplied by solar panel must be $0.5 \mathrm{~V}$ higher than that of rechargeable battery because of the forward voltage drop of diode. Whenever the charging voltage is below the threshold, the diode disconnects the solar panel from the battery. As confirmed by our experimental results, in bad weather e.g., cloudy, the harvested energy cannot be transmitted to the rechargeable battery since the charging voltage is not high enough. Different from the environmental noise, this problem may disable the entire network the whole day or even longer. A possible approach is to enlarge the size of solar panel in order to collect more environmental energy even though the radiating rate is low, or replace the current Li-ion battery by a larger one. However, it will cause extra hardware cost which makes it unpractical in large scale sensor network. In our work, we try to solve the above problems by substituting the diode-based circuit with a maximum power point tracker system(MPPT). Such design requires the development of an adaptive system to transfer the energy generated by the solar panel into a storage medium, such as Li-ion battery or a ultra-capacitor. Existing MPPT [9] circuit consists of a buck and boost DC/DC converter and its peripheral circuit. The DC/DC converter is introduced here because it can convert a low voltage to a relatively high voltage in order to provide sufficient charging voltage. This is a feasible option without considering the loss in charging efficiency. However, since the charging efficiency by DC/DC is lower than direct charging which will be explained later, then how to increase the efficiency becomes a hidden challenge.

In this paper, we propose a brand new charging circuit by (1) 


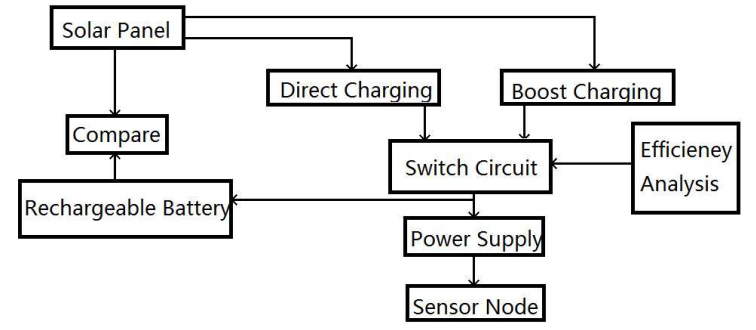

Fig. 4. Flow Diagram

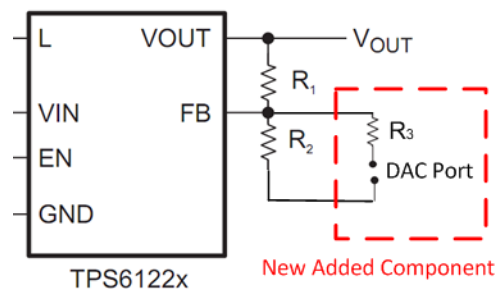

Fig. 5. Modified Architecture of TPS61220. replacing diode with triode; (2) substituting a high efficiency low input voltage step-up converter for a regular buck and boost DC/DC converter; and (3) combining this converter with triode. We have two different charging modes in our system: direct-charging and boost-charging mode. We connect the collector to the anode of solar panel, the base to the GPIO on TelosB, and the emitter to the anode of Li-ion battery.

Direct-charging: when the charging voltage is high enough to support direct charging, we let the system stay at directcharging mode where the MCU on the TelosB pull the base to low, the current will be led from solar panel to Li-ion battery directly, and the energy can be stored in the Li-ion battery easily. When the charging stops, the MCU pull the base to high, so that the current between the solar panel and Li-ion battery will be cut off, and the back flow can be avoided.

Boost-charging: when the environmental condition does not allow the direct charging, e.g., the charging voltage is below the threshold, the system will switch to boost-charging mode where we lead the current to the boost DC/DC converter. After boosting to a higher voltage, we can charge the battery.

Depending on different weather and charging conditions, our system will switch between these two modes automatically. Figure 4 illustrate how our system works.

We next discuss the charging efficiency of our system. The charging efficiency is defined as the ratio between harvested energy and environmental energy. Empirical study shows that the direct-charging mode provides the highest charging efficiency, since there is no energy loss theoretically. Note that when the input voltage is very low, although the output voltage can be boosted very high, the real energy will be taken a great discount from original one.

Although direct charging has the best charging efficiency, this charging mode often suffers from its own drawbacks as mentioned earlier, e.g., the charging voltage may not be high enough. Remember that in diode-based system, the voltage offered by the solar panel must be $0.5 \mathrm{~V}$ higher than that of rechargeable battery. As the voltage of rechargeable battery increases, the required charging voltage from solar panel also increases. If the charging voltage is not high enough, we can not harvest the energy anymore, where the energy can be considered to be wasted. Thus we have a trade-off between direct charging and boost charging here.

Software-defined Charging Voltage. Regarding this tradeoff, if we can dynamically adjust the output voltage to $e x$ - actly $0.5 \mathrm{~V}$ higher than the current voltage of rechargeable battery, we can guarantee that the charging voltage is high enough while ensuring the charging efficiency. In order to achieve this objective, a step-up DC/DC converter (TPS61220) is introduced in the boost-charging mode. Traditionally, the output voltage is programmed by an external resistor divider, once the resistor divider and feedback voltage $V_{F B}$ is fixed, the output voltage $V_{O U T}$ is also determined which can be calculated by: $R_{1}=R_{2} \cdot\left(V_{O U T} / V_{F B}-1\right)$. In order to adaptively adjust the output voltage $V_{O U T}$, we have two possible options here: 1) adjust $V_{F B} ; 2$ ) adjust the value of $R_{1}$ or $R_{2}$. Since the feedback voltage $V_{F B}$ is almost fixed(around $0.5 \mathrm{~V}$ ) which can not be controlled by us, thus we concentrate on the second option. At the first sight, it seems also hopeless since both $R_{1}$ and $R_{2}$ are physically fixed and can hardly be adjusted dynamically. We next design a software-defined charging voltage system by adding a new hardware component. Basically, the new component contains a new resistor $R_{3}$ which is connected with the DAC prot on TelosB. Please refer to Fig. 5 for illustration. Then we may treat the component consisting of $R_{2}, R_{3}$ and TelosB as the original $R_{2}$. By dynamically changing the output of DAC port, we can further control the output voltage based on the above equation. In the boost-charging mode, the node will monitor the voltage of rechargeable battery in real time, and calculate the required charging voltage, and produce desired output through DAC port.

Software Design. We next briefly describe the software designed for our platform. The efficiency analysis mechanism is introduced to evaluate the optimal efficiency based on the current condition, such as weather, supplying voltage and remaining energy. Energy information is passed by applications to SmartMote through defined APIs, and processed later by the compare mechanism. ADC12 port is used to sample the realtime voltage supplied by both environment and Li-ion battery as introduced earlier. Then the charging mode can be decided immediately, and the relevant triodes are triggered by pulling the base to low by the GPIO on SmartMote. The triodes can be regarded as switches here, through which the SmartMote can switch between different charging modes dynamically.

\section{Network PlanNing}

In the previous section, we introduce the architecture of our platform. In this section, we will concentrate on designing the 


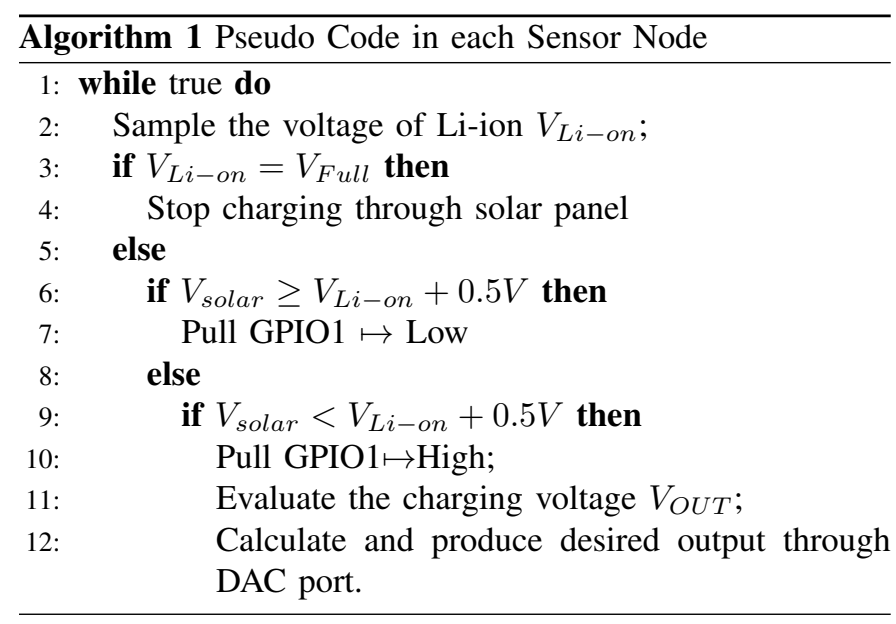

network planning strategy. In particular, our network planning strategy for each period is composed of two parts:

1) We present a maximum weighted energy allocation scheme for each sensor, and flow control scheme which cooperates with our energy allocation. Specifically, we first study how to balance the harvested energy between sensing and transmission for each sensor in order to achieve maximum weight. Accordingly, an associated flow allocation scheme for each link has been proposed. Since our scheme repeatedly determines both the direction and quantity of the flow over each link, the routing strategy is not required;

2) Further, by taking possible interference into consideration, we implement an interference-free link scheduling strategy in order to minimize the transmission latency.

In the real experiment, since we often need to collect the raw data from all sensors for further research, data collection acts as a dominate operation in the network. Thus, we will focus on the data collection case where no data aggregation or fusion is allowed in this work. Remember that we will estimate the amount of harvested energy at the beginning of each period of 45 minutes. Then based on the estimation results, we further find the network planning strategy for this period. We formally define the problem as follows:

Network Planning Problem: Given a set of $n$ sensor nodes and $m$ sink nodes with their locations, the estimated amount of harvested energy $E_{i}$ of sensor $i$ during current period and the weight $w_{i}$ associated with $i$, we need to find a maximum weighted network planning strategy for this period in order to collect all the sensed data to the sink nodes.

Let $f_{i, j}$ denote the total number of packets that node $i$ transmits to node $j$, and $s_{i}$ denote the total amount of sensed data generated by node $i$ over the this period. Note that all the $s_{i}$ packets of sensing data need to be transmitted to one of the sink nodes. Since any valid schedule must respect the energy constraints at each sensor, it follows that for each sensor $i$,

$$
\sum_{j=1}^{n+1} f_{i, j} \cdot T_{X_{i, j}}+\sum_{j=1}^{n} f_{j, i} \cdot R_{X_{i}}+s_{i} \cdot S_{X_{i}} \leq E_{i}
$$

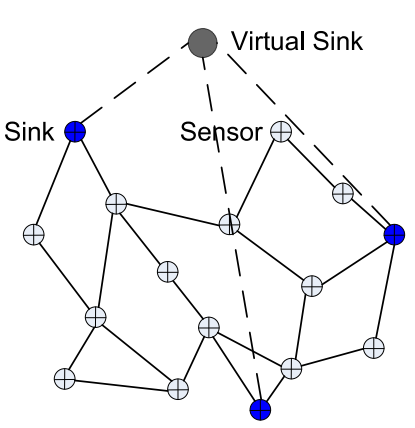

(a)

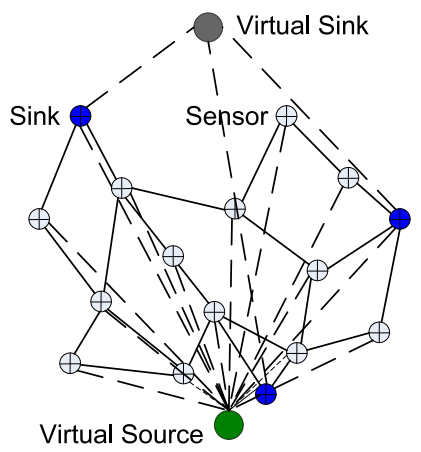

(b)
Fig. 6. (a) After virtual sink is added; (b) After virtual source is added.

\section{A. Resource Allocation}

We first study how to balance the harvested energy between sensing and transmission for each sensor. We further investigate how to allocate the flow to each link in order to achieve maximum total weight $\sum w_{i} \cdot s_{i}$. Clearly, a desired scheduling for each period comprises two parts: 1) the amount of sensed data $s_{i}$ generated at each sensor node $i ; 2$ ) the flow allocation on each link. We say a flow network is feasible if and only if it obeys both energy constraint and flow conservation. A feasible flow network with maximum weight is called an optimal feasible flow network.

As an illustration shown in Fig. 6, the dark nodes represent the sink nodes, the light nodes denote the sensor nodes and any adjacent nodes can communicate with each other. Obviously, we have multiple sensor nodes and sink nodes in the original problem which is extremely complicated to solve directly. To reduce the great complexity derived from the original problem, we introduce a virtual sink to the original network. As illustrated in Fig. 6(a), we add a link with length 0 between the virtual sink and each sink node. We further assign weight 0 to each sink node. In the new constructed graph, our objective is to find a energy/flow allocation strategy for each sensor and link to collect all sensing data to the virtual sink. To this end, we have successfully converted the original multisink problem to a single-sink problem. And the energy/flow allocation computed from above can be directly applied to the original network by ignoring the virtual sink.

We next concentrate on finding a scheduling on the new constructed graph. An optimal feasible flow network can be found using the following integer program with linear constraints. We define the following variables in the integer program: $f_{i, j}$ denotes the capacity over link $(i, j) ; b$ pakcets/second denotes the bandwidth of each link, $s_{i}$ packets/period denotes the sensing rate of sensor $i$; $s_{\max }$ denotes the maximum sensing rate; $f_{\max }$ denotes the maximum transmission rate per period over one link, which can be defined as $b \times 45 \times 60$; for each sensor $k=1,2, \cdots, n$, let $\pi_{i, j}^{(k)}$ be a flow variable indicating the amount of packets that sensor $k$ sends to the virtual sink $n+1$ over link $(i, j)$. The integer program is given by

$$
\max \sum w_{i} s_{i}
$$




$$
\begin{cases}\text { a) } \quad \sum_{j=1}^{n} \pi_{j, i}^{(k)}=\sum_{j=1}^{(n+1)} \pi_{i, j}^{(k)}, \\ & \forall i=1,2, \cdots, n \text { and } i \neq k \\ \text { b) } \quad s_{k}+\sum_{j=1}^{n} \pi_{j, k}^{(k)}=\sum_{j=1}^{n+1} \pi_{k, j}^{(k)} \\ c) \quad 0 \leq \sum_{k=1}^{n} \pi_{i, j}^{(k)} \leq f_{i, j} \\ & \forall i=1, \cdots, n \forall j=1 \cdots, n+1 \\ \text { d) } \quad \sum_{i=1}^{n} \pi_{i, n+1}^{(k)}=s_{k} \\ \text { e) } \quad 0 \leq s_{k} \leq s_{\max }, 0 \leq f_{i, j} \leq f_{\max }\end{cases}
$$

where all the variables are required to take integer values. For each $k=1,2, \cdots, n$, constraint (5) consists of following restrictions: $a$ ) and $b$ ) ensures the flow conservation principle at each sensor; $c$ ) ensures that the capacity constraints on each link are respected; $d$ ) ensures that all sensing data $s_{k}$ generated from sensor $k$ can reach the virtual sink and $e$ ) ensures that the maximum sensing rate and transmission rate constraints are satisfied.

When all the variables are allowed to take fractional values, the linear relaxation of the above integer program can be computed in polynomial-time. Then, we can obtain an approximation for the feasible flow network as follows.

1) First, we fix the sensing rate $s_{i}$ to the floor of their values obtained from the linear relaxation for each sensor $i$. In this way, the energy constrains are all satisfied. And the reduction on the weighted achieved with respect to the optimal solution is at most $\sum_{i=1}^{n} w_{i}$.

2) Next, by solving the maximum flow problem, we can get the flow allocation on each link. Specifically, we introduce a virtual source to the graph as illustrated Fig. 6(b). And we add a edge between the virtual source node and any other sensor node $i$ (except for the virtual sink), with capacity $s_{i}$. We further define the capacity of each edge between virtual sink and original sink node as infinity. The capacity of any original edge $(i, j)$ is set to $f_{i, j}$ as computed above. Then by considering the virtual source as source node and virtual sink as target node, we solve the maximum $s$ - $t$ flow problem on the new constructed graph. Finally, the flow allocation on each edge can be derived from the above solution.

So far, we already find the energy allocation between sensing and transmitting for each sensor. And the routing strategy can also be derived from the flow allocation immediately.

\section{B. Interference-free Link Scheduling}

Based on the discussion in the previous section, we can decide the amount of packets (flow) allocated to each link over one period. Next we try to find an interference-free link scheduling in order to minimize the overall data transmission latency. Basically, we schedule each link in a TDMA manner. We divide one period (45 minutes) into $45 \times 60 / 5$ rounds with length 5 seconds for each round. Since we already obtain the amount of packets transmitted over each link during one period, we can immediately get the average demand on each link per round. Then we implement the following link scheduling strategy round by round. Our interference-free link scheduling can be modeled as a Minimum Fractional Weighted

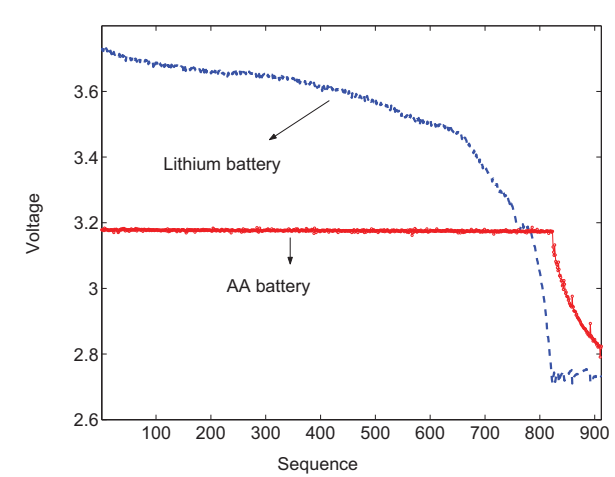

Fig. 8. The discharging pattern on our dual power system.

Link Schedule (MFWLS) Problem, which is proved to be NPHard in [10]. In this paper, we use the scheduling method proposed in [11] to achieve low latency link scheduling.

\section{EXPERIMENT RESULTS}

To evaluate the performance of our platform and network planning strategy, we implement them on the two testbeds as described in Section II. Basically, we first test the charging and discharging pattern of our system under different scenarios using the smaller testbed. We further conduct a network-wide experiment, using the larger tesdbed, to monitor the topology of entire network and find that the network topology may vary under different environment. Finally, we test the network planning strategy in the larger testbed and our experimental results show that the overall VoI achieved by strategy is very close to the optimal one.

\section{A. Charging Pattern Measurement}

Outdoor Environment: We conducted experiments to find different charging patterns under different weather conditions. The experiment is launched at 21:55:51 GMT+08:00, July 16, 2009. 2009, and ended at 19:54:59 GMT+08:00, July 17, 2009. The experimental results are shown in Fig. 7(a), the charging pattern is presented as the voltage vs. luminous intensity. We only reported the pattern of one node here due to space limit although we measured the patterns of much more sensors. The experimental results confirm our assumption that the charging voltage varies significantly within one day.

Indoor Environment: This evaluation is deployed under the lamp in our lab. Compared with the outdoor scenario, the luminous intensity dramatically decrease, and the energy harvested here is extremely limited. However, the advantage of indoor charging is that the intensity of the light is stable where the reliability can be guarantee. The experimental results are shown in Fig.7(b).

\section{B. Discharging Pattern Measurement on Dual Power System}

We also test the discharging pattern on the dual power system. The duty cycle is set to $10 \%$, with all LEDs on. Initially, the energy of both Li-ion and two AA batteries are on their full capacity, the energy of Li-ion battery is $1700 \mathrm{mAh}$, 


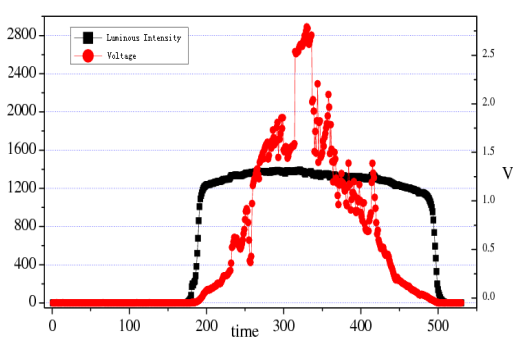

(a)

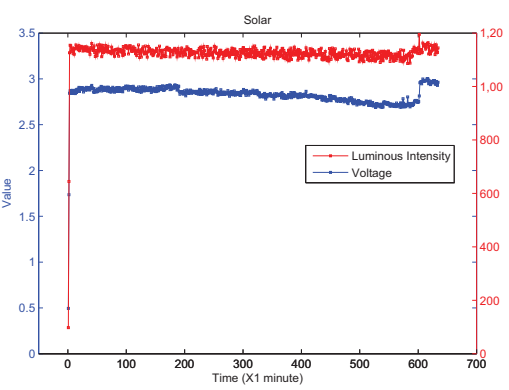

(b)

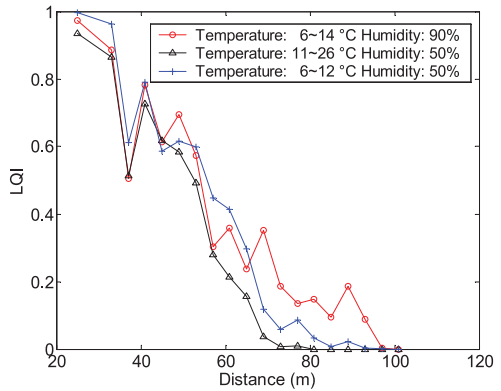

(c)

Fig. 7. (a)The charging pattern measured in outdoor environment; (b)The charging pattern measured in indoor environment; (c) [12]LQI under varying temperature and humidity.

while the two AA batteries are $3000 \mathrm{mAh}$, the curves are the voltage of these two batteries when they are discharging. The voltage of the Li-ion drops gradually as providing energy to the node at the first few hours, and then, it stages a dramatically decrease when the energy is nearly exhausted. When the voltage of $\mathrm{Li}$-ion reaches $2.7 \mathrm{~V}$, the peripheral circuit automatically switches the input power from $\mathrm{Li}$-ion to AA battery. As shown in Fig.8, the voltage of AA battery remains stable until the voltage Li-ion battery reaches $2.7 \mathrm{~V}$.

\section{Environment-sensitive Link Quality and Network Topology}

Since our system is deployed in the forest, we are able to find the impact of various environmental factors, e.g., humidity and temperature, on our system. Here we use the Link Quality Indicator (LQI) [7] as a metric to measure the wireless link quality, which is then measured under varying temperatures and humidity. Figure 7(c) plots LQI between two nodes as a function of node distance in three different scenarios, where the temperature and humidity differ from one another.

\section{Evaluation of Network Planning Strategy}

We implement our network planning strategy on the real testbed to evaluate its performance in terms of average weight of collected information. In particular, we adopted a monitoring application that collects the humidity information for research on forest. By setting different weight to different sensors, we monitored the average weighted of collected information by different strategies throughout one day. In order to obtain a upper bound on the weight of optimum solution, we solve the liner programming in Section VI-A directly. In addition, we introduce another routing strategy, called Fixed Routing Strategy(FRS), basically, each sensor always sends its data to its closest sink through shortest path. The main results are demonstrated in Fig.9. The average weight by our strategy can achieve the maximum value around 2:00PM since the average amount of harvested energy is maximized at that time. Clearly, the performance of our strategy is very close to the optimum solution throughout one day. One the other hand, it performs much better and stable than FRS.

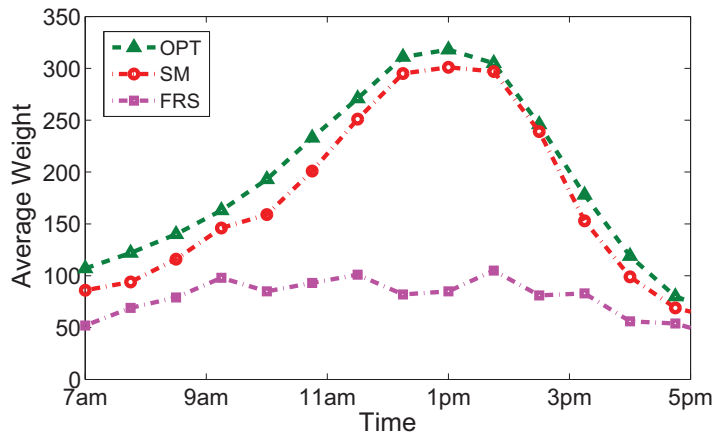

Fig. 9. The achieved weight by SmartMote(SM) is almost same as the optimum solution while much better than the Fixed Routing Strategy.

\section{RELATED WORK}

Energy scarcity is, however, a first class design concern for a large body of research in wireless sensor networks. Low-power hardware platforms with energy harvesting support [13]-[15] as well as algorithms for estimating and predicting energy harvesting and consumption [9], [15], [16] are crucial components of all perpetual systems. Additionally, a variety of energy-aware networking techniques have been proposed for use with low-power sensors, including energy- aware clustering [17], aggregation, and traffic shaping to extend device lifetimes [18].

Solar-powered wireless sensor network attracts more and more attention these days. Jay et al. design a micro-solar power subsystems for wireless sensor nodes, Alippi et al. [9] proposed a low-energy maximum power point tracker (MPPT) which is designed to optimize the use of solar energy. Very recently, Yang et al. [19] a reliable storage service, called SolarStore, that adaptively trades-off storage reliability versus energy consumption in solar-powered sensor networks. Wang et al. [20] propose a reliable data collection and storage system for solar-powered sensor networks. Many works have taken energy into consideration when design network planning strategy, e.g., routing, link scheduling. However, most of existing approaches did not consider the various charging pattern at different time. Meanwhile, many researchers study 
the coverage problem in perpetual network(such as solar [21], [22], wind [23], and thermal [24]). Basically, they try to find a wake-up scheduling for each sensor under the energy constraint in order to achieve good coverage quality.

\section{CONCLUSION}

In this paper, we present the SmartMote system, which balances sensing with packet delivery for an energy harvesting sensor network. We design a Dual Power Supply system which enables our system work in a more stable way. We further design an enhanced platform which is able to harvest the energy even under harsh environment. Accordingly, we propose a set of network planning strategies in order to collect as much valuable data as possible. We finally conduct a set of experiments on the real test bed to evaluate our system. Currently, we are working on deploying a few hundred sensors for environment monitoring.

\section{ACKNowledgement}

The research of authors is partially supported by NSF CNS0832120, NSF CNS-1035894, National Basic Research Program of China (973 Program) under grant No. 2010CB328101, 2010CB334707, 2011CB302705, National Natural Science Foundation of China under Grant No. 61170216, Tsinghua National Laboratory for Information Science and Technology (TNList), program for Zhejiang Provincial Key Innovative Research Team, and program for Zhejiang Provincial Overseas High-Level Talents (One-hundred Talents Program).

\section{REFERENCES}

[1] K. Kar, A. Krishnamurthy, and N. Jaggi, "Dynamic node activation in networks of rechargeable sensors," in Proceedings IEEE INFOCOM 2005. 24th Annual Joint Conference of the IEEE Computer and Communications Societies, vol. 3, 2005.

[2] M. Tacca, P. Monti, and A. Fumagali, "Cooperative and noncooperative ARQ protocols for microwave recharged sensor nodes," in Wireless Sensor Networks, 2005. Proceeedings of the Second European Workshop on, pp. 45-56.

[3] A. Kansal, D. Potter, and M. Srivastava, "Performance aware tasking for environmentally powered sensor networks," in Proceedings of the joint international conference on Measurement and modeling of computer systems. ACM New York, NY, USA, 2004, pp. 223-234.

[4] T. Banerjee, S. Padhy, and A. Kherani, "Optimal Dynamic Activation Policies in Sensor Networks," in Communication Systems Software and Middleware, 2007. COMSWARE 2007. 2nd International Conference on, 2007, pp. 1-8.

[5] V. Pryyma, L. Bölöni, and D. Turgut, "Uniform sensing protocol for autonomous rechargeable sensor networks," in MSWiM 08'. ACM New York, NY, USA, 2008, pp. 92-99.

[6] M. Gatzianas, L. Georgiadis, and L. Tassiulas, "Asymptotically optimal policies for wireless networks with rechargeable batteries," in Wireless Communications and Mobile Computing Conference, 2008. IWCMC'08. International, 2008, pp. 33-38.

[7] J. Polastre, R. Szewczyk, and D. Culler, "Telos: Enabling ultra-low power wireless research,' in IPSN '05. IEEE Press, 2005, p. 48.

[8] W. Heinzelman, A. Chandrakasan, and H. Balakrishnan, "Energy efficient Communication Protocols for Wireless Microsensor Networks," in Proc. of Hawaiian International Conference on Systems Science, 2000.

[9] A. Kansal, J. Hsu, S. Zahedi, and M. Srivastava, "Power management in energy harvesting sensor networks," ACM Transactions on Embedded Computing Systems (TECS), vol. 6, no. 4, p. 32, 2007.

[10] L. C. and Y. M., "On the hardness of approximating minimization problems," in Journal of ACM.
[11] P. Wan, "Multiflows in multihop wireless networks," in Proceedings of the tenth ACM international symposium on Mobile ad hoc networking and computing. ACM, 2009, pp. 85-94.

[12] Y. L. J. Z. S. T. X.-Y. L. L. Mo, Y. He and G. Dai, "Canopy closure estimates with greenorbs: Sustainable sensing in the forest," in Proceedings of SenSys'09. ACM, 2009.

[13] K. Lin, J. Yu, J. Hsu, S. Zahedi, D. Lee, J. Friedman, A. Kansal, V. Raghunathan, and M. Srivastava, "Heliomote: enabling long-lived sensor networks through solar energy harvesting," in Proceedings of the $3 r d$ international conference on Embedded networked sensor systems. ACM, 2005, p. 309.

[14] X. Jiang, J. Polastre, and D. Culler, "Perpetual environmentally powered sensor networks," in IPSN '05. IEEE Press, 2005, p. 65

[15] J. Sorber, A. Kostadinov, M. Garber, M. Brennan, M. Corner, and E. Berger, "Eon: a language and runtime system for perpetual systems," in Proceedings of the 5th international conference on Embedded networked sensor systems. ACM, 2007, p. 174.

[16] A. Dunkels, F. Osterlind, N. Tsiftes, and Z. He, "Software-based on-line energy estimation for sensor nodes," in Proceedings of the 4th workshop on Embedded networked sensors. ACM, 2007, p. 32.

[17] O. Younis and S. Fahmy, "HEED: a hybrid, energy-efficient, distributed clustering approach for ad hoc sensor networks," IEEE Transactions on Mobile Computing, pp. 366-379, 2004.

[18] C. Schurgers and M. Srivastava, "Energy efficient routing in wireless sensor networks," in MILCOM, vol. 1. Citeseer, 2001, pp. 357-361.

[19] Y. Yang, L. Wang, D. Noh, H. Le, and T. Abdelzaher, "SolarStore: enhancing data reliability in solar-powered storage-centric sensor networks," in Proceedings of the 7th MobiSys. ACM New York, NY, USA, 2009, pp. 333-346.

[20] L. Wang, D. Noh, Y. Yang, H. Le, T. Abdelzaher, and M. Ward, "AdaptSens: An adaptive data collection and storage service for solarpowered sensor networks," Urbana, vol. 51, p. 61801.

[21] J. Hsu, A. Kansal, J. Friedman, V. Raghunathan, and M. Srivastava, "Energy Harvesting Support for Sensor Network," Proc. of IEEE IPSN Demo, 2005.

[22] V. Raghunathan, A. Kansal, J. Hsu, J. Friedman, and M. Srivastava, "Design considerations for solar energy harvesting wireless embedded systems," in IIPSN 2005., 2005, pp. 457-462.

[23] C. Park and P. Chou, "Ambimax: Autonomous energy harvesting platform for multi-supply wireless sensor nodes," 2006.

[24] I. Stark, "Invited talk: Thermal energy harvesting with Thermo Life," in Wearable and Implantable Body Sensor Networks, 2006. BSN 2006. International Workshop on, 2006, pp. 19-22. 